\title{
Marker for Real-Time Analysis of Caspase Activity in Intact Cells
}

BioTechnqiues 33:1284-1291 (December 2002)

\author{
Pui Lee, Elaine Beem, and \\ Mark S. Segal \\ University of Florida, \\ Gainesville, FL, USA
}

\begin{abstract}
Apoptosis, or programmed cell death, is an important regulator of growth, development, defense, and homeostasis in multicellular organisms. A family of cysteine proteases known as caspases is central to many apoptotic pathways, and thus detection of their activity offers an effective means to assess apoptosis. However, currently available methods only allow the evaluation of in vivo caspase activity at a given time point or over a few hours. To assess the activity over extended periods of time, we designed a novel, real-time, in vivo marker that utilizes the $N$ end rule degradation pathway to allow the detection of caspase activity as reflected by increasing enhanced GFP (EGFP) stability. The marker has an N-terminal arginine in the absence of caspase activity and is rapidly degraded. In vivo caspase activity removes the marker's N-terminal arginine residue, leaving an EGFP with an $\mathrm{N}$-terminal methionine that results in stable fluorescence. In our study, the marker accurately depicted an increase in caspase activity in apoptotic cells and also detected significant endogenous caspase activity in non-apoptotic cells. The downstream effects of this endogenous activity detected in intact, nonapoptotic cells must be regulated by the cell preventing apoptosis. These studies also demonstrate the feasibility of using the $N$ end rule to study endogenous enzymatic activities other than those associated with proteasomal degradation.
\end{abstract}

\section{INTRODUCTION}

Apoptosis, or programmed cell death, is an important regulator of growth, development, defense, and homeostasis in multicellular organisms $(17,33)$. A family of cysteine proteases known as caspases is central to many apoptotic pathways (29). Caspases cleave at the carboxyl end of a four amino acid consensus site that ends in aspartic acid $(28,31)$. In non-apoptotic cells, the inactive precursor forms of these enzymes, known as procaspases, are constitutively expressed. Procaspases are activated by the proteolytic removal of the prodomain and the formation of heterodimers $(13,18,20)$.

There are two classes of caspases involved in apoptosis. The initiator caspases-2, $-8,-9$, and -10 propagate and perhaps amplify the apoptotic signal (20). The executioner caspases-3, -6, and -7 are responsible for cleaving cytoskeleton regulatory proteins (i.e., gelsolin and focal adhesion kinase), nuclear structures (lamins), DNA repair proteins $\left(\mathrm{DNA}-\mathrm{PK}_{\mathrm{cs}}\right)$, and the inhibitors of DNases (Icad/DFF45) (20,25, $31)$. The destruction or deregulation of these and other cellular targets culminates in the morphological and chemical characteristics of apoptosis. Since caspases are important mediators of the process, measurements of their activity offer a great tool to detect programmed cell death $(26,37)$.

The most common way to assess caspase activity during apoptosis is to use fluorometric or colorimetric substrates. Such substrates are made by conjugating a quencher compound via a specific caspase cleavage site to a fluorophore or chromophore. Caspase cleavage frees the fluorophore or chromophore from the quencher molecule, allowing for detection by fluorescence or light emission $(23,37)$. Another popular method uses a biotinylated irreversible caspase inhibitor that binds to active caspase and is detected by immunoblot with streptavidin conjugates (14).

Recent reports suggest that procaspases have inherent caspase cleavage activity $(12,14,24)$. However, currently available methods only allow the evaluation of in vivo caspase activity at a given time point or over a few hours and may not accurately reflect this endogenous activity. To accurately assess caspase activity in non-apoptotic cells over hours or days, we created a novel, realtime, in vivo marker. The design of this marker utilizes the $\mathrm{N}$-end rule degradation pathway to allow the detection of caspase activity as reflected by increasing the stability of a recombinant enhanced GFP (EGFP). The rate of protein degradation via proteasomes is determined by their N-terminal amino acid (1). Proteins that have an N-terminal methionine are long-lived in comparison with proteins with an N-terminal arginine (32). Previously, the N-end rule has been used to examine proteasomal activity $(8,9,32)$; however, we have applied the $\mathrm{N}$-end rule to study caspase activity. The marker we designed will have an N-terminal arginine in the absence of caspase activity or an N-terminal methionine in the presence of caspase activity within the cell. We have used this marker to analyze caspase activity in non-apoptotic and apoptotic cells. 


\section{MATERIALS AND METHODS}

\section{Materials}

All primers and tissue culture reagents were obtained from Invitrogen (Carlsbad, CA, USA), enzymes were obtained from PanVera (Madison, WI, USA), and all other reagents were obtained from Sigma (St. Louis, MO, USA) unless otherwise noted.

\section{Plasmid Construction}

Using a ubiquitin sequence-containing plasmid (a kind gift from Dr. Stewart Lecker, Beth Israel Deaconess Medical Center, Boston, MA) as a template, PCR was performed with the primers 5'-AACCCAAGCTTATGCAGATCT-

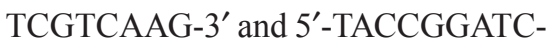
CTCGACCACCTCTTAGTCTTAAG3'. The second PCR used pEGFP-C1 plasmid (BD Biosciences Clontech, Palo Alto, CA, USA) as a template with the primers 5'-TTAGGATCCTTAGAAACAGACATGGTGAGCAAGGGCG-3' and 5'-TGGGAATTCTCACTTGTACAGCTCGTCC- $3^{\prime}$. The control constructs were created by substituting the methionine codon, arginine codon, or both for the underlined codon in the above primers.

The ubiquitin PCR product and the pIND plasmid (Invitrogen) were digested with the restriction enzymes EcoRI/BamHI and ligated by standard molecular biology methods (22). The resultant plasmid and the pEGFP PCR product were digested with the restriction enzymes BamHI/NotI and ligated. The marker plasmids were verified by DNA sequencing.

\section{Cell Culture}

HEK 293T cells were cultured in DMEM with $10 \%$ FBS, $100 \mu \mathrm{g} / \mathrm{mL}$ penicillin, and $100 \mathrm{mg} / \mathrm{mL}$ streptomycin at $37^{\circ} \mathrm{C}$ with $5 \%(\mathrm{v} / \mathrm{v}) \mathrm{CO}_{2}$.

\section{Tranfection and Induction}

Cells previously plated in $60-\mathrm{mm}$ dishes $\left(1 \times 10^{6}\right.$ cells/plate $)$ were transfected with $4 \mu \mathrm{g}$ marker plasmid, $4 \mu \mathrm{g}$ VgRXR plasmid (Invitrogen), and $1 \mu \mathrm{g}$ cytomegalovirus (CMV)- $\beta$-galactosidase plasmid by the calcium phosphate method (22). The VgRXR plasmid directs expression of the ecdysone receptor, making cells responsive to Ponasterone A (Invitrogen). Exogenous caspase activity was introduced by co-transfection with the pIND-CD8-Caspase-8 (from $50 \mathrm{ng}$ to $1 \mu \mathrm{g} /$ transfection) (A kind gift from Richard Siegel, National Institutes of Health, Bethesda, MD), and all cells were transfected with an equal amount of DNA. Twenty-four hours after transfection, the cells were induced with $5 \mu \mathrm{M}$ Ponasterone A for $24 \mathrm{~h}$.

\section{Immunoblotting}

Cells $\left(2 \times 10^{6}\right)$ were lysed in $50 \mu \mathrm{L}$ Reporter Lysis Buffer (Promega, Madison, WI, USA), resolved along with broad range molecular weight markers (Bio-Rad Laboratories, Hercules, CA, USA) by SDS-PAGE, and transferred to nitrocellulose membrane as previously described (24). The membrane was blocked in $5 \%$ milk, probed with 1:1000 rabbit anti-GFP (Santa Cruz Biotechnologies, Santa Cruz, CA, USA), and washed in TBS ( $\mathrm{pH}$ 7.4) $/ 0.1 \%$ Tween ${ }^{\circledR} 20$ before probing with 1:25000 HRP-conjugated goat anti-rabbit (Zymed, San Francisco, CA, USA). West-Dura Enhanced Chemiluminescence (Pierce Chemical, Rockford, IL, USA) was added for detection by Fluorchem Imaging System (Alpha InnoTech, San Leandro, CA, USA).

\section{FACS $^{\circledR}$ Analysis}

Cells $\left(5 \times 10^{5}\right)$ from the collected samples were suspended in $0.5 \mathrm{~mL}$ PBS with or without $5 \mu \mathrm{g} / \mathrm{mL}$ propidium iodide (Molecular Probes, Eugene, OR, USA). The cells were sorted by FAC$\mathrm{Scan}^{\mathrm{TM}}$, and the data were analyzed with CellQuest software (both from BD Biosciences, San Jose, CA, USA).

\section{Fluorescence Quantitation}

After $24 \mathrm{~h}$ of Ponasterone A induction, the cells were collected and assayed with $\beta$-Galactosidase Enzyme Assay System (Promega) using a PowerWave 200 (Bio-Tek Instruments, Winooski, VT, USA). Simultaneous fluorescence was measured in a FL 600 Fluorescent Microplate Reader (BioTek Instruments).

\section{RESULTS}

\section{Caspase Marker Design}

We designed a marker with the ability to continuously monitor the activity of caspases within intact cells (Figure 1A). The marker, R-LETD-M-EGFP, relies on the enzymatic activity of two families of proteases (ubiquitin hydrolases and caspases) and the N-end rule of degradation. Ubiquitin hydrolase, which cleaves co-translationally after ubiquitin with no regard to the subsequent amino acid, is used to produce a protein with an $\mathrm{N}$-terminal arginine, which is targeted for rapid proteasomal degradation by the $\mathrm{N}$-end rule (Figure 1B). Caspase cleavage after the consensus sequence lysine-glutamate-threonine-aspartic acid (LETD) eliminates the N-terminal arginine and produces a protein with an $\mathrm{N}$-terminal methionine (Figure 1B). Proteins with N-terminal methionine are more stable than their arginine counterparts, which are degraded via the proteasomal pathway at a rate at least 100 -fold slower.

To evaluate the in vivo behavior of the marker, R-LETD-M-EGFP, and to determine whether marker synthesis overwhelms the cleavage and degradation pathways, three control markers were constructed (Figure 1C). The RLETD-R-EGFP construct produces a protein with an $\mathrm{N}$-terminal arginine after ubiquitin cleavage and after cleavage of the LETD by caspases. Cells transfected with this plasmid should have minimal fluorescence unless protein production overwhelms degradation pathways, and the fluorescence pattern should not be affected by the presence of caspase activity (Figure 1C). Conversely, M-LETD-M-EGFP serves as a positive control since the construct will yield a protein with an $\mathrm{N}$ terminal methionine after ubiquitin cleavage and after cleavage of the LETD by caspases. Cells transfected with this plasmid should have maximum fluorescence unaffected by the presence of caspase activity (Figure 1C). Cells transfected with the MLETD-R-EGFP construct should exhibit maximal and minimal fluorescence under opposite conditions compared with cells transfected with the R-LETD-M-EGFP marker: stable 
fluorescence after ubiquitin cleavage but rapid degradation after caspase cleavage leaves an N-terminal arginine (Figure 1C).

\section{Marker Behavior in Apoptotic Cells}

We assessed the changes in total fluorescence of cells expressing $\mathrm{M}$ LETD-R-EGFP and R-LETD-MEGFP upon co-transfection with the CD8-caspase-8, a plasmid encoding a membrane-bound form of active caspase- 8 (11). The amount of the caspase- 8 plasmid transfected into cells was titrated to prevent overwhelming apoptosis. When low amounts of the caspase- 8 plasmid are co-transfected into cells with M-LETD-R-EGFP or RLETD-M-EGFP, cellular fluorescence decreased by more than $50 \%$ or increased by $62 \%$, respectively (Figure 2 ). These reciprocal changes suggest that expression of caspase- 8 results in increased cleavage at the LETD site that yields M-EGFP and R-EGFP proteins. The R-EGFP protein has a shorter half-life than M-EGFP, resulting in decreased fluorescence. Although the fluorescence of the cells decreased with the addition of caspase- 8 , the cells expressing the R-LETD-M-LETD marker consistently demonstrated higher fluorescence than the cells expressing the M-LETD-R-LETD marker (Figure 2).

\section{Transfection and EGFP Expression in Eukaryotic Cells}

To confirm that the cells transfected with the markers expressed the expected EGFP recombinant protein, immunoblot analysis was performed. No EGFP protein greater than $29.7 \mathrm{kDa}$ was detected in any transfected cells, which is consistent with nearly complete cleavage of the ubiquitin sequence (Figure 3). Surprisingly, the cells expressing M-LETD-M-EGFP contained two bands that have EGFP reactivity; one band migrated with an apparent molecular weight of $29.7 \mathrm{kDa}$, the expected size of EGFP with an intact M-LETD sequence, and another band migrated with an apparent molecular weight of $29.1 \mathrm{kDa}$, the expected size of M-EGFP. This suggested that caspase activity was present in the cells transfected with the plasmids (Figure
$3)$. The 1:4 ratio of caspase cleaved $(29.1 \mathrm{kDa})$ to uncleaved $(29.7 \mathrm{kDa})$ EGFP indicated that the observed caspase activity was significant. While both cleaved and uncleaved products were expected for other constructs, those with $\mathrm{N}$-terminal arginine should degrade rapidly.

To confirm that all of the expressed proteins could be cleaved by caspases and to evaluate the molecular weight of all construct proteins in the absence and presence of exogenous caspase, we cotransfected the cells with a plasmid encoding for active caspase- 8 in a membrane-bound form (11). Indeed, only the 29.1-kDa recombinant proteins, either M-EGFP or R-EGFP, were detected after the introduction of active caspase-8 (Figure 3).
Only one band of $29.3 \mathrm{kDa}$ with EGFP reactivity was present in cells transfected with M-LETD-R-EGFP. Co-transfection with the caspase- 8 plasmid suggested that this $29.3-\mathrm{kDa}$ band contained the LETD sequence because, in the presence of caspase- 8 , only a smaller $29.1-\mathrm{kDa}$ band was detected that migrated similarly to EGFP. Although we saw two bands in the cells transfected with the M-LETD-MEGFP, the cells transfected with $\mathrm{M}$ LETD-R-EGFP produced only one EGFP-reactive band. No 29.1-kDa protein was detected in cells transfected with M-LETD-R-EGFP in the absence of exogenous caspase- 8 , perhaps because cleavage of the LETD sequence produced the protein R-EGFP, which has a short half-life. R-EGFP was de-

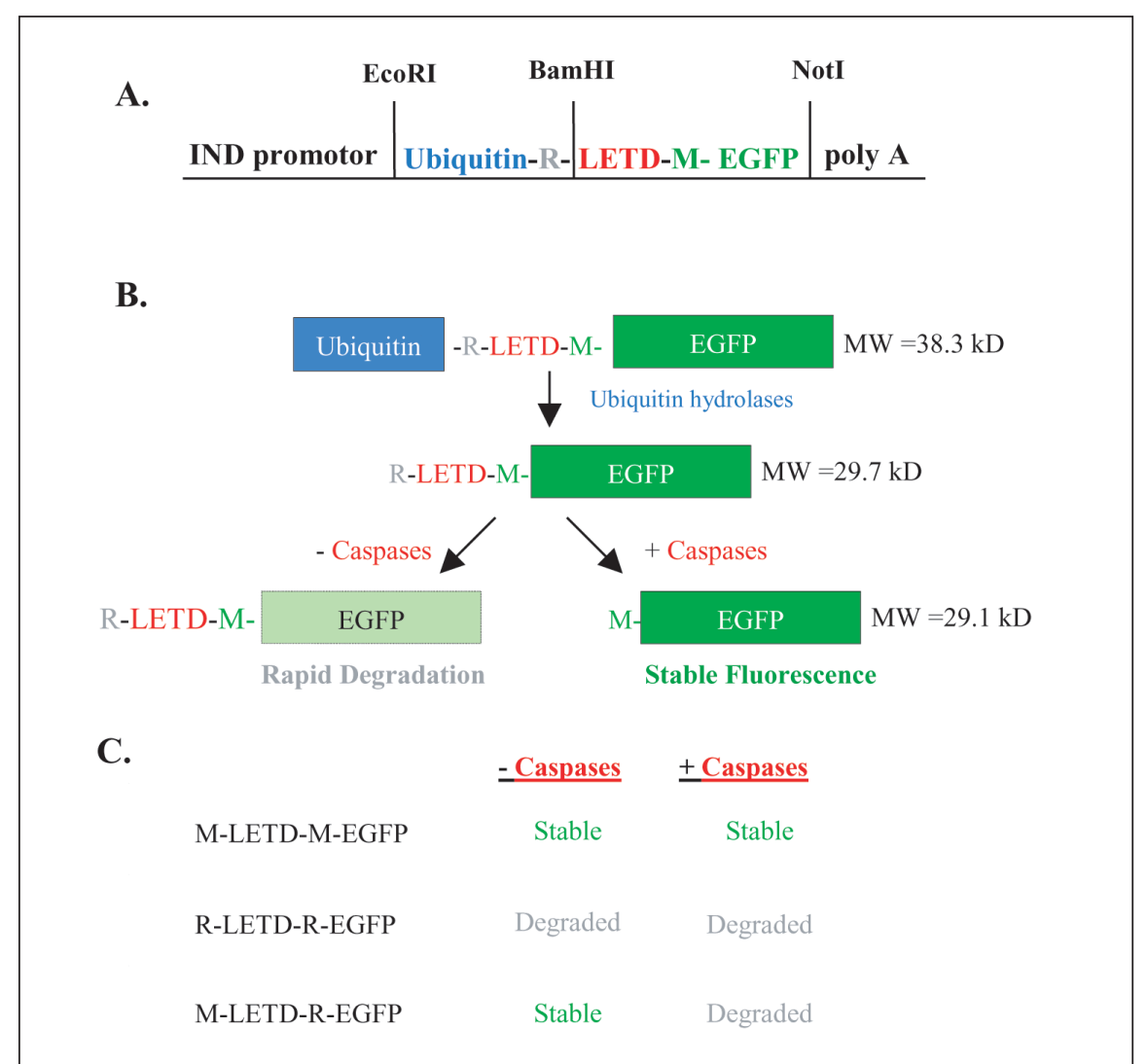

Figure 1. Design of the in vivo caspase marker R-LETD-M-EGFP. (A) The linear representation of the marker illustrates that the inducible promoter pIND drives the production of the recombinant protein that consists of the 76 amino acid ubiquitin sequence, followed sequentially in frame by an arginine, the caspase cleavage site LETD, and EGFP with a N-terminal methionine. Restriction sites used to construct the plasmid are shown. (B) Upon expression of the marker, ubiquitin hydrolases co-translationally cleaves after the ubiquitin sequence, leaving an N-terminal arginine on the recombinant EGFP. Subsequent cleavage after the consensus sequence LETD by caspase activity eliminates the N-terminal arginine and leaves an EGFP with an N-terminal methionine. (C) The control constructs differ from RLETD-M-EGFP by only one or two amino acid codons, and only the cDNA after the ubiquitin sequence is displayed. The stability of the fluorescence produced by transfection of the construct into cells in the absence and presence of caspase activity is indicated. 
tected when cells were transfected with M-LETD-R-EGFP and the caspase-8 plasmid, perhaps because proteasomal degradation is overwhelmed.

Whether or not caspase- 8 is co-expressed in the cells transfected with RLETD-M-EGFP, only a 29.1-kDa EGFP is detected. N-terminal arginine present on proteins not cleaved by caspases may lead to the rapid degradation of the protein, thus only the more stable M-EGFP is detected. Any protein expressed by R-LETD-R-EGFP should degrade rapidly regardless of caspase activity; therefore, the faint band detected likely represents proteins not yet degraded by proteasomes. It is interesting that the band detected by immunoblot in cells transfected with RLETD-R-EGFP in the absence of exogenous caspase- 8 migrates with an apparent molecular weight of $29.2 \mathrm{kDa}$ while in the presence of exogenous caspase- 8 , the detected band migrates with an apparent molecular weight of 29.1 $\mathrm{kDa}$. This suggests that the $29.2-\mathrm{kDa}$ protein contains an intact LETD sequence. The inability to detect a 29.1 $\mathrm{kDa}$ band in the absence of the caspase8 plasmid may be due to the overall poor stability of the N-terminal arginine proteins and the very low amount of the 29.1-kDa protein present without exogenous caspase- 8 . The unexpected

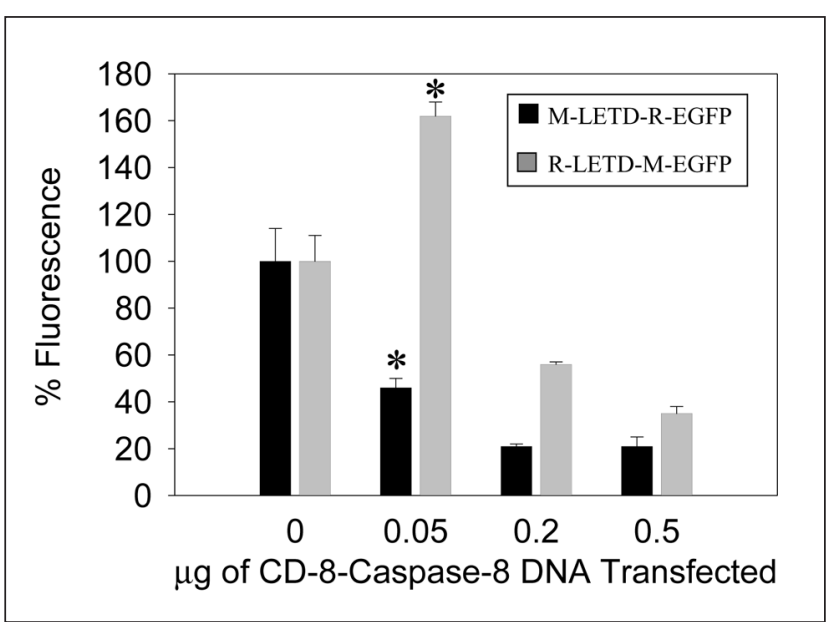

Figure 2. Caspase activity results in increased fluorescence in the cells expressing R-LETD-M-EGFP. CD8-caspase-8 plasmid ( $0-0.5 \mu \mathrm{g})$ was co-transfected together with $4 \mu \mathrm{g}$ of either M-LETD-R-EGFP (black bars) or R-LETD-M-EGFP (gray bars). One day after transfection, the cells were induced for $24 \mathrm{~h}$ with Ponasterone A before cell lysate collection. Fluorescence was normalized for protein concentration and is expressed as a percentage of fluorescence obtained from cells lacking the caspase- 8 plasmid. Results are $\overline{\mathrm{x}} \pm$ SEM of triplicate samples. An asterisk indicates a statistical difference $(P<0.05)$ compared to $0 \mu \mathrm{g}$ transfected CD8-caspase-8 plasmid. pase activity within the transfected cells then we would expect that the cells transfected with M-LETD-R-EGFP would fluoresce similar to the cells transfected with M-LETD-M-EGFP, and the cells transfected with R-LETDM-EGFP would fluoresce similar to the cells transfected with R-LETD-REGFP. Differences in fluorescence were not attributable to a specific effect of the transfection procedure since a similar pattern was seen even when the induction was delayed for $72 \mathrm{~h}$ after transfection (data not shown). This result was consistent with the immunoblot results and supported the interpretation that either a small percentage of the cells were apoptotic or that there was endogenous caspase activity present in non-apoptotic cells.

To distinguish between these two possibilities, FACS was used to evaluate the fluorescence distribution in the cells transfected with the plasmids (Figure 5). The cells transfected with each marker fell into a normal distribution of fluorescence. As expected, the cells expressing M-LETD-M-EGFP had the highest mean value of fluorescence while the cells expressing RLETD-R-EGFP had the lowest value of fluorescence. Unexpectedly, the MLETD-R-EGFP and R-LETD-MEGFP populations showed nearly identical distributions of fluorescence, with a mean fluorescence in between the means of M-LETD-M-EGFP and RLETD-R-EGFP. This population pattern was not just the result of induction 
of apoptosis by transfection since gating only propidium iodide-negative cells yielded a similar distribution (data not shown).

\section{DISCUSSION}

Caspases are involved in many apoptotic pathways. All of the currently available caspase assays examine the activity of the enzyme at a specific point in time and do not allow a kinetic evaluation over hours to days. We developed a marker capable of evaluating caspase activity for an extended period of time. The design of this marker exploits the properties of both ubiquitin hydrolases and the N-end rule of protein degradation, with caspase activity reflected by increasing the stability of EGFP.

Recently, GFP was reported to be an effective $\mathrm{N}$-end rule substrate as $\mathrm{R}$ GFP degraded significantly faster than M-GFP $(2,3)$. In our study, proteolytic removal of ubiquitin from the construct proteins by ubiquitin proteases was evident by the size of the detected EGFP. The pattern of fluorescence was consistent with the N-end rule: cells transfected with R-LETD-R-GFP fluoresced significantly less than those expressing

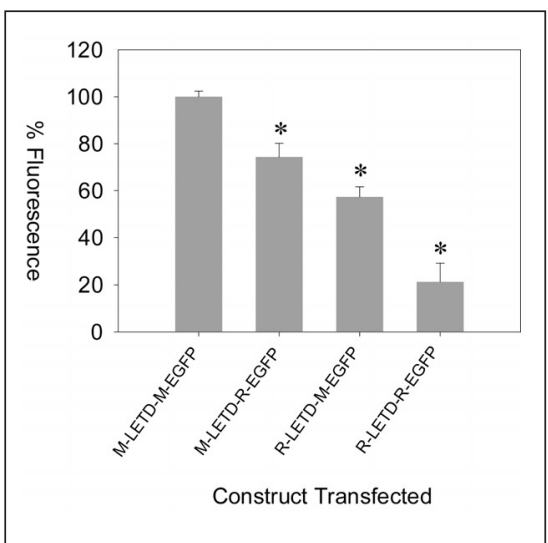

Figure 4. Comparison of total fluorescence in the cells expressing marker and control constructs. One day after cells were transfected with $4 \mu \mathrm{g}$ construct plasmid, Ponasterone A was added to the media for $24 \mathrm{~h}$ before cell lysate collection. Fluorescence was normalized for $\beta$-galactosidase activity and is expressed as a percentage of fluorescence obtained from the cells transfected with the M-LETD-M-EGFP construct plasmid. Results are $\bar{X} \pm$ SEM of triplicate samples and are representative of five independent experiments. An asterisk indicates a statistical difference $(P<0.05)$ compared to $0 \mu \mathrm{g}$ M-LETD-M-EGFP.
M-LETD-M-GFP. In our expression system, a methionine residue only stabilized the protein 5 -fold as compared with an arginine residue (Figure 4), which is consistent with the findings previously reported for GFP (2).

In non-apoptotic cells, we assumed that there would be little or no caspase activity. However, by immunoblot, we detected two bands in the cells expressing M-LETD-M-EGFP (Figure 3). The lower band migrated with the same molecular weight as the single EGFP band detected when caspase- 8 was coexpressed in cells. This suggests that caspase activity is present in non-apoptotic cells or that a portion of the transfected cells is apoptotic. Because the population of the cells analyzed by FACS was a normal distribution and the pattern did not change after gating propidium iodide-negative cells, it is unlikely that the immunoblot results are attributable to a small population of apoptotic cells. This is supported by the identical distribution of the fluorescence of cells transfected with RLETD-M-EGFP and M-LETD-REGFP. If only a small portion of cells was responsible for the apparent caspase activity then one would expect that cells transfected with either RLETD-M-EGFP or M-LETD-R-EGFP would have a bimodal distribution, which was not seen.
It is also unlikely that the transfection itself or induction with Ponasterone A activated caspase activity. Nearly identical results were obtained from the cells transfected by lipid reagents or calcium phosphate and when Ponasterone A induction was delayed for three days after transfection (data not shown). Ponasterone A was not responsible for the induction of caspase activity since both the $29.7-\mathrm{kDa}$ and 29.1-kDa EGFP were detected from the expression of M-LETD-MEGFP under the constitutive promoter pCMV for up to five days after transfection (data not shown). This indicates that the detected caspase activity was independent of exogenous agents and likely to be endogenous in origin.

We have avoided specifying which caspase is responsible for the endogenous caspase activity even though we have used the consensus sequence for caspase-8, LETD, in the construction of the plasmid. Many reports of overlapping specificities are in the literature $(28,30)$, thus we cannot determine whether one caspase or many different caspases are responsible for this activity. However, it is apparent that this endogenous caspase activity is not just present in 293T cells since experiments performed with Cos-7 cells demonstrated similar results (data not shown).

The endogenous caspase activity in

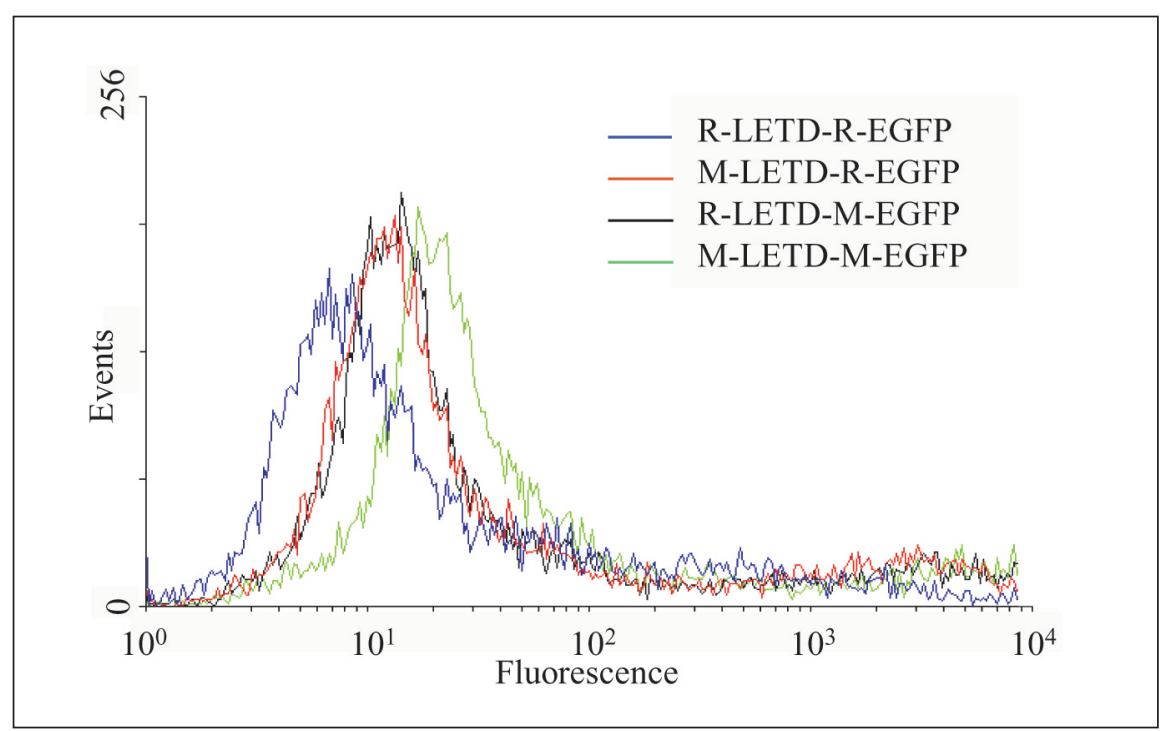

Figure 5. FACS of cells expressing marker and control constructs. One day after cells were transfected with $4 \mu \mathrm{g}$ construct plasmid, Ponasterone A was added to the media for $24 \mathrm{~h}$ before cell fixation with $4 \%$ paraformaldehyde. Ten thousand cells were analyzed by FACS. The results are representative of 10 independent experiments. 
non-apoptotic cells can originate either from active caspases that are found during apoptosis or from their precursors, procaspases. The latter possibility is consistent with previous reports that some procaspases contain $1 \%-10 \%$ of the proteolytic activity found in active enzymes $(14,24)$. Such small but detectable procaspase activity is the basis for the induced proximity model of caspase- 8 activation (14). The model suggests that clustering of procaspase- 8 molecules accelerates the trans-cleavage and autoactivation of caspases by the inherent proteolytic activity of procaspases. Perhaps the same explanation also applies for the detection of active caspases from bacterial expressions of recombinant procaspases (27).

Compartmentalization of procaspases, their cofactors, and substrates plays a major role in the regulation of apoptosis, and the precise localization of procaspases is controversial $(6,16,19,21,38)$.
However, compartmentalization cannot completely account for what prevents the low but detectable activity within cells from cleaving cellular substrates and activating other caspases in nonapoptotic cells. Cleavage of our construct proteins by caspase activity without inducing apoptosis further suggests the existence of regulatory mechanisms to tightly control the proteolytic activity.

Endogenous caspase inhibitors, such as inhibitors of apoptosis proteins (IAPs) have recently attracted considerable attention and may be central to this regulatory system $(7,34)$. It is clear that IAPs inhibit active caspases, but the extent that IAPs inhibit endogenous caspase activity of procaspases is unknown $(4,36)$. Perhaps the endogenous activity we detect is not inhibitable by IAPs; however, the downstream effect of this endogenous activity, the production of active caspases, is inhibited by IAPs, thus preventing apoptosis. In- deed, this endogenous activity of procaspases may be vital for the initiation of programmed cell death. For apoptosis to proceed, IAPs would need to be inhibited by smac/diablo (5), other inhibitors, and/or overwhelming activation of caspase $3(7,10,34,35)$.

Since the marker is dependent on a number of different protease activities, including proteasomal degradation, it will be used in future studies to determine the rate of proteasomal degradation during apoptosis. In addition, we have established the feasibility of using the N-end rule as the basis of an assay for the enzymatic activity of an enzyme other than those involved in proteasomal degradation.

\section{ACKNOWLEDGMENTS}

We would like to thank S. Gluck and members of the Gluck laboratory for 
many helpful discussions. This work was financially supported by National Institutes of Health grant no. DK02537 and Gatorade Research funds (M.S.S.). The authors wish to thank the staff of the Flow Cytometry Core Laboratory at the University of Florida's Interdisciplinary Center for Biotechnology Research, for assistance with flow cytometry.

\section{REFERENCES}

1.Bachmair, A., D. Finley, and A. Varshavsky. 1986. In vivo half-life of a protein is a function of its amino-terminal residue. Science 234:179-186.

2.Dantuma, N.P., S. Heessen, K. Lindsten, M. Jellne, and M.G. Masucci. 2000. Inhibition of proteasomal degradation by the gly-Ala repeat of Epstein-Barr virus is influenced by the length of the repeat and the strength of the degradation signal. Proc. Natl. Acad. Sci. USA 97:8381-8385.

3.Dantuma, N.P., K. Lindsten, R. Glas, M. Jellne, and M.G. Masucci. 2000. Short-lived green fluorescent proteins for quantifying ubiquitin/proteasome-dependent proteolysis in living cells. Nat. Biotechnol. 18:538-543.

4.Deveraux, Q.L., H.R. Stennicke, G.S. Salvesen, and J.C. Reed. 1999. Endogenous inhibitors of caspases. J. Clin. Immunol. 19:388-398

5.Du, C., M. Fang, Y. Li, L. Li, and X. Wang. 2000. Smac, a mitochondrial protein that promotes cytochrome c-dependent caspase activation by eliminating IAP inhibition. Cell 102:33-42.

6.Ghribi, O., M.M. Herman, and J. Savory. 2002. The endoplasmic reticulum is the main site for caspase-3 activation following aluminum-induced neurotoxicity in rabbit hippocampus. Neurosci. Lett. 324:217-221.

7.Holcik, M., H. Gibson, and R.G. Korneluk. 2001. XIAP: apoptotic brake and promising therapeutic target. Apoptosis 6:253-261.

8.Levy, F., N. Johnsson, T. Rumenapf, and A. Varshavsky. 1996. Using ubiquitin to follow the metabolic fate of a protein. Proc. Natl. Acad. Sci. USA 93:4907-4912.

9.Levy, F., J.A. Johnston, and A. Varshavsky. 1999. Analysis of a conditional degradation signal in yeast and mammalian cells. Eur. J. Biochem. 259:244-252

10.MacFarlane, M., W. Merrison, S.B. Bratton, and G.M. Cohen. 2002. Proteasome-mediated degradation of Smac during apoptosis: XIAP promotes Smac ubiquitination in vitro. J. Biol. Chem. 277:36611-36616.

11.Martin, D.A., R.M. Siegel, L. Zheng, and M.J. Lenardo. 1998. Membrane oligomerization and cleavage activates the caspase- 8 (FLICE/MACHalpha1) death signal. J. Biol. Chem. 273:4345-4349.

12.Muzio, M. 1998. Signaling by proteolysis: death receptors induce apoptosis. Int. J. Clin. Lab. Res. 28:141-147.

13.Muzio, M., G.S. Salvesen, and V.M. Dixit. 1997. FLICE induced apoptosis in a cell-free system. Cleavage of caspase zymogens. J.
Biol. Chem. 272:2952-2956.

14.Muzio, M., B.R. Stockwell, H.R. Stennicke, G.S. Salvesen, and V.M. Dixit. 1998. An induced proximity model for caspase- 8 activation. J. Biol. Chem. 273:2926-2930.

15.Noel, D., K. Nikaido, and G.F. Ames. 1979 A single amino acid substitution in a histidinetransport protein drastically alters its mobility in sodium dodecyl sulfate-polyacrylamide ge electrophoresis. Biochemistry 18:4159-4165.

16.Qin, Z.H., Y. Wang, K.K. Kikly, E. Sapp, K.B. Kegel, N. Aronin, and M. DiFiglia. 2001. Pro-caspase- 8 is predominantly localized in mitochondria and released into cytoplasm upon apoptotic stimulation. J. Biol. Chem. 276:8079-8086.

17.Raff, M. 1998. Cell suicide for beginners. Nature 396:119-122.

18.Renatus, M., H.R. Stennicke, F.L. Scott R.C. Liddington, and G.S. Salvesen. 2001 Dimer formation drives the activation of the cell death protease caspase 9. Proc. Natl. Acad. Sci. USA 98:14250-14255.

19. Ritter, P.M., A. Marti, C. Blanc, A. Baltzer, S. Krajewski, J.C. Reed, and R. Jaggi. 2000. Nuclear localization of procaspase-9 and processing by a caspase-3-like activity in mammary epithelial cells. Eur. J. Cell Biol. 79:358-364.

20.Salvesen, G.S. and V.M. Dixit. 1997. Caspases: intracellular signaling by proteolysis. Cell 91:443-446.

21.Samali, A., B. Zhivotovsky, D.P. Jones, and S. Orrenius. 1998. Detection of pro-caspase3 in cytosol and mitochondria of various tissues. FEBS Lett. 431:167-169.

22.Sambrook, J. and D.W. Russell. 2001. Molecular Cloning :A Laboratory Manual. CSH Laboratory Press, Cold Spring Harbor, NY.

23.Segal, M.S. and E. Beem. 2001. Effect of pH, ionic charge, and osmolality on cytochrome cmediated caspase-3 activity. Am. J. Physiol. Cell Physiol. 281:C1196-C1204.

24.Stennicke, H.R., Q.L. Deveraux, E.W. Humke, J.C. Reed, V.M. Dixit, and G.S Salvesen. 1999. Caspase-9 can be activated without proteolytic processing. J. Biol. Chem. 274:8359-8362.

25.Stennicke, H.R., J.M. Jurgensmeier, H. Shin, Q. Deveraux, B.B. Wolf, X. Yang, Q. Zhou, H.M. Ellerby, et al. 1998. Pro-caspase-3 is a major physiologic target of caspase-8. J. Biol. Chem. 273:27084-27090.

26.Stennicke, H.R. and G.S. Salvesen. 2000 Caspase assays. Methods Enzymol. 322:91100.

27.Stennicke, H.R. and G.S. Salvesen. 2000 Caspases - controlling intracellular signals by protease zymogen activation. Biochim. Biophys. Acta 1477:299-306.

28.Talanian, R.V., C. Quinlan, S. Trautz, M.C. Hackett, J.A. Mankovich, D. Banach, T. Ghayur, K.D. Brady, et al. 1997. Substrate specificities of caspase family proteases. J. Biol. Chem. 272:9677-9682.

29.Thornberry, N.A. and Y. Lazebnik. 1998 Caspases: enemies within. Science 281:1312 1316.

30.Thornberry, N.A., T.A. Rano, E.P. Peterson, D.M. Rasper, T. Timkey, M. GarciaCalvo, V.M. Houtzager, P.A. Nordstrom, et al. 1997. A combinatorial approach defines specificities of members of the caspase family and granzyme B. Functional relationships established for key mediators of apoptosis. J. Biol. Chem. 272:17907-17911.

31.Troy, C.M. 2001. Diversity of caspase involvement in neuronal cell death, p. 67-91. In M.P. Mattson, S. Estus, and V.M. Rangnekar (Eds.), Programmed Cell Death Volume 1Cellular and Molecular Mechanisms. Elsevier, Baltimore, MD

32.Varshavsky, A. 1996. The N-end rule: functions, mysteries, uses. Proc. Natl. Acad. Sci. USA 93:12142-12149.

33.Vaux, D.L. and A. Strasser. 1996. The molecular biology of apoptosis. Proc. Natl. Acad. Sci. USA 93:2239-2244.

34.Verhagen, A.M., E.J. Coulson, and D.L. Vaux. 2001. Inhibitor of apoptosis proteins and their relatives: IAPs and other BIRPs. Genome Biol 2:REVIEWS3009.

35.Verhagen, A.M. and D.L. Vaux. 2002. Cell death regulation by the mammalian IAP antagonist Diablo/Smac. Apoptosis 7:163-166.

36.Villa, P., S.H. Kaufmann, and W.C. Earnshaw. 1997. Caspases and caspase inhibitors. Trends Biochem. Sci. 22:388-393.

37.Zhang, G., V. Gurtu, C. Spencer, J.-T. Ma, and S.R. Kain. 1998. Detection of caspase activity associated with apoptosis using fluorimetric and colorimetric methods, p. 7-14. In L. Zhu and J. Chun (Eds.), Apoptosis Detection and Assay Methods. Eaton Publishing, Westborough, MA.

38.Zhivotovsky, B., A. Samali, A. Gahm, and S. Orrenius. 1999. Caspases: their intracellular localization and translocation during apoptosis. Cell Death Differ. 6:644-651.

Received 21 August 2002; accepted 7 October 2002

Address correspondence to:

Dr. Mark S. Sega

P.O. Box 100224

Gainesville, FL 32610, USA

e-mail:segalms@medicine.ufl.edu

For reprints of this or any other article, contact Reprints@BioTechniques.com 